\title{
Some Calculations Related to Riemann's Prime Number Formula
}

\author{
By Hans Riesel and Gunnar Göhl
}

Abstract. The objective of this paper is to study the relation of the complex zeros of the Riemann zeta function to the distribution of prime numbers. This relation arises from a formula of Riemann, which is studied here by extensive machine calculations. To establish the validity of the computations, reasonable upper bounds for the various errors involved are deduced. The analysis makes use of a formula, (32), which seems to be quite new.

Only the first 29 pairs of complex zeros $\rho=\frac{1}{2} \pm i \alpha(\alpha<100)$, and the primes in the interval $x<10^{6}$ are considered. It turns out that these zeros of $\zeta(s)$ lead to an approximation of $\pi(x)$, the number of primes $\leqq x$, that gives the integer part correctly up to about $x=1000$.

Introduction. Following Ingham [4, p. 82], we define

$$
\operatorname{li}\left(e^{u-i n}\right)=\int_{-\infty+i v}^{u+i v} e^{z} d z / z \quad(v \neq 0 \text { or } c=0 \text { and } u<0)
$$

and

$$
\operatorname{li}(x)=\lim _{\delta \rightarrow+0}\left(\int_{0}^{1-\delta}+\int_{1+\delta}^{x}\right) \frac{d t}{\log t} \quad(x>1) .
$$

We then set

$$
\pi_{0}(x)=\frac{1}{2}\{\pi(x+0)+\pi(x-0)\} .
$$

In 1859 Riemann [1] published, and in 1895 von Mangoldt [2] proved, the following formula:

$$
\pi_{0}(x)=\sum_{n=1}^{\infty} \mu(n) f\left(x^{1 / n}\right) / n,
$$

where $\mu(n)$ is the Möbius function, and

$$
f(x)=\sum_{n=1}^{\infty} \pi_{0}\left(x^{1 / n}\right) / n=1 \mathrm{i}(x)-\sum_{\rho} \operatorname{li}\left(x^{\rho}\right)+\int_{x}^{\infty} \frac{d t}{\left(t^{2}-1\right) t \log t}-\log 2,
$$

where the sum means $\lim _{T \rightarrow \infty} \sum_{|\rho| \leqq T} \operatorname{li}\left(x^{\rho}\right)$, and the $\rho$ 's are the nontrivial zeros of the Riemann zeta function:

$$
\zeta(s)=\sum_{n=1}^{\infty} n^{-s}=\prod_{p}\left(1-p^{-s}\right)^{-1} .
$$

In the sum over the $\rho$ 's, each $\rho$-term appears a number of times equal to the multiplicity of the zero $\rho$. We remark also that, since $f(x)=0$ for $1<x<2$, the sum

Received May 22, 1968, revised March 16, 1970.

AMS 1969 subject classifications. Primary 1041, 1042, 1043; Secondary 6525.

Key words and phrases. Riemann's prime number formula, zeros of Riemann zeta function, Gram's series. 
in (4) is actually finite, and equals

$$
\pi_{0}(x)=\sum_{n=1}^{N} \mu(n) f\left(x^{1 / n}\right) / n
$$

for all $x<2^{x+1}$, because then $x^{1 / n}<2$ for all $n \geqq N+1$, and so $f\left(x^{1 / n}\right)=0$. We shall always choose $N$, so that $2^{N+1}>x$.

Taking only the first term $\mathrm{li}(x)$ of (5), and introducing it into the "inversion formula" (4), Riemann got his famous approximation to $\pi_{0}(x)$ :

$$
\pi_{0}(x) \approx R(x)=\sum_{n=1}^{\infty} \mu(n) \operatorname{li}\left(x^{1 / n}\right) / n .
$$

The right-hand side of (8) can be transformed into Gram's series (see [5])

$$
R(x)=1+\sum_{n=1}^{\infty} \frac{(\log x)^{n}}{n ! n \zeta(n+1)} .
$$

The Complex Part of Riemann's Formula. Since

$$
\frac{d^{k} R\left(e^{t}\right)}{d t^{k}}=\sum_{n=k}^{\infty} \frac{t^{n-k}}{(n-k) ! n \zeta(n+1)}>0 \quad \text { for } t>0,
$$

it is obvious that $R(x)$ cannot describe the more detailed behaviour of $\pi_{0}(x)$, which is certainly not a function with all derivatives $>0 . R(x)$ smoothes the values of $\pi_{0}(x)$ and gives a kind of meanvalue correct smooth approximation to $\pi_{0}(x)$. The lack of a more detailed agreement with $\pi_{0}(x)$ is due to the fact that only the first term in (5) was taken into account in (8). Thus we can state that the other terms in (5), especially the infinite series, are responsible for the more detailed behaviour of $\pi_{0}(x)$. Since the inversion formula (4) is linear in $f$, it is possible to examine the influence on $\pi_{0}(x)$ by each separate term in (5).

Having made these observations, the following problem might be suggested:

To study how the value of $\pi_{0}(x)$ is related to each pair of complex conjugate zeros $\rho$ and $\bar{\rho}$ of $\zeta(s)$, beginning with the first pair of zeros, $\rho=\frac{1}{2} \pm 14.134725 i$. It seemed feasible to the authors to make such a study, at least for reasonably large values of $x$ and $\rho$, if the necessary numerical calculations were done on a computer.

Qualitative Results for Large Values of $x$. If one integrates (1) by parts, one obtains

$$
\int_{-\infty+i r}^{u+i v} \frac{e^{z} d z}{z}=\left[\frac{c^{\xi}}{z}\right]_{-\infty+i v}^{u+v}+\int_{-\infty+i r}^{u+i r} \frac{e^{z} d^{\prime} z}{z^{2}},
$$

and an easy estimate gives

$$
\left|\frac{\int_{-\infty}^{u+i v} \frac{e^{z} d z}{z^{2}}}{\frac{e^{u+i v}}{u+i v}}\right| \rightarrow 0
$$

as $u+i v$ tends to $\infty$ along any fixed ray not the positive real axis. Thus, as $u+i v$ tends to $\infty$ along such a ray,

$$
\operatorname{li}\left(e^{u+i v}\right) \sim \frac{e^{u+i,}}{u+i v} .
$$


Thus, for $\rho$ a root of $\zeta(s)$,

$$
\operatorname{li}\left(x^{\rho}\right)=\operatorname{li}\left(e^{\rho \log x}\right) \sim \frac{e^{\rho \log x}}{\rho \log x},
$$

where $\rho \log x$ moves along a fixed ray, as $x \rightarrow \infty$, and this ray is not the positive real axis. Hence for a root lying on the critical line, $\rho=\frac{1}{2}+i \alpha$, we have

$$
\begin{aligned}
-\left\{\operatorname{li}\left(x^{\rho}\right)+\operatorname{li}\left(x^{\bar{\rho}}\right)\right\} & =-2 \operatorname{Re} \operatorname{li}\left(x^{\rho}\right) \sim-2 \operatorname{Re} \frac{\sqrt{ } x e^{i \alpha \log x}}{\left(\frac{1}{2}+i \alpha\right) \log x} \\
& =\frac{-2 \sqrt{ } x}{|\rho| \log x} \cdot \cos (\alpha \log x-\arg \rho) .
\end{aligned}
$$

Thus, for $x$ large, the contribution to $\pi_{0}(x)$ from two complex conjugate zeros $\frac{1}{2} \pm i \alpha$ of $\zeta(s)$ is an oscillating function with an amplitude varying with $x$ as $2 \sqrt{ } x /(|\rho| \log x)$ and with the consecutive zeros $x_{k+1}$ and $x_{k}$ connected by the relationship $x_{k+1}=$ $x_{k} \cdot e^{\pi / \alpha}$. These functions are shown for the first 5 pairs of zeros in Fig. 1. The larger $|\rho|$ becomes, the smaller is the amplitude, and the faster are the oscillations.

Quantitative Results Obtained by Computations. For $x=2(1) 100$ computations of

$$
T_{k}(x)=-\sum_{n=1}^{N} \frac{\mu(n)}{n}\left\{\operatorname{li}\left(x^{\rho_{k} / n}\right)+\operatorname{li}\left(x^{\bar{\rho}_{k} / n}\right)\right\}
$$

were carried out for each of the first 29 pairs of zeros of $\zeta(s)$, for which $\alpha<100$. These values of $T_{k}(x)$ were used in (7) to give the approximations $R_{k}(x), k=0$, $1,2, \cdots, 29$, of $\pi_{0}(x)$ for $x<2^{N+1}$ :

$$
R_{k}(x)=\sum_{n=1}^{N} \mu(n) f_{k}\left(x^{1 / n}\right) / n,
$$

where

$$
f_{k}(x)=\operatorname{li}(x)-\sum_{|\rho| \leq|\rho| \rho \mid} \operatorname{li}\left(x^{\rho}\right)+\int_{x}^{\infty} \frac{d t}{\left(t^{2}-1\right) t \log t}-\log 2 .
$$

The different values of $R_{k}(x)$ were compared to $\pi_{0}(x) . f_{0}(x)$ is meant as (16) without any term $\operatorname{li}\left(x^{\rho}\right)$.

In the results of these computations, the oscillating character of $T_{k}(x)$, discussed in the preceding section, is easily recognizable. Compare Fig. 1 with Fig. 2, which shows $T_{k}(x)$ for $k=1(1) 5$. It is also, by comparison with $\pi_{0}(x)$, easily seen, to what extent just a few pairs of zeros of $\zeta(s)$ exhaust the difference between $\pi_{0}(x)$ and Riemann's approximation (8). This comparison is shown in Figs. 3a-d for different numbers of zeros and for different intervals. The intervals chosen in Figs. $3 \mathrm{c}$ and $\mathrm{d}$ show the behaviour in the neighbourhood of a comparatively large interval, containing only one prime in the middle, namely the interval $(200,222)$ with the prime 211 , and the largest prime-free interval below 1000 , which is $(888,906)$. It turns out that the approximations "in mean" are getting better and better when the number of zeros taken into account increases, but the new approximations are not much superior to Riemann's, unless $x$ is comparatively small. This can be explained by the fact that the first pair of zeros has such a large distance from the origin $\left(\rho_{1}=\right.$ $\left.\frac{1}{2}+14.13 i\right)$, and that the following zeros then follow close to the first. Thus Riemann's 

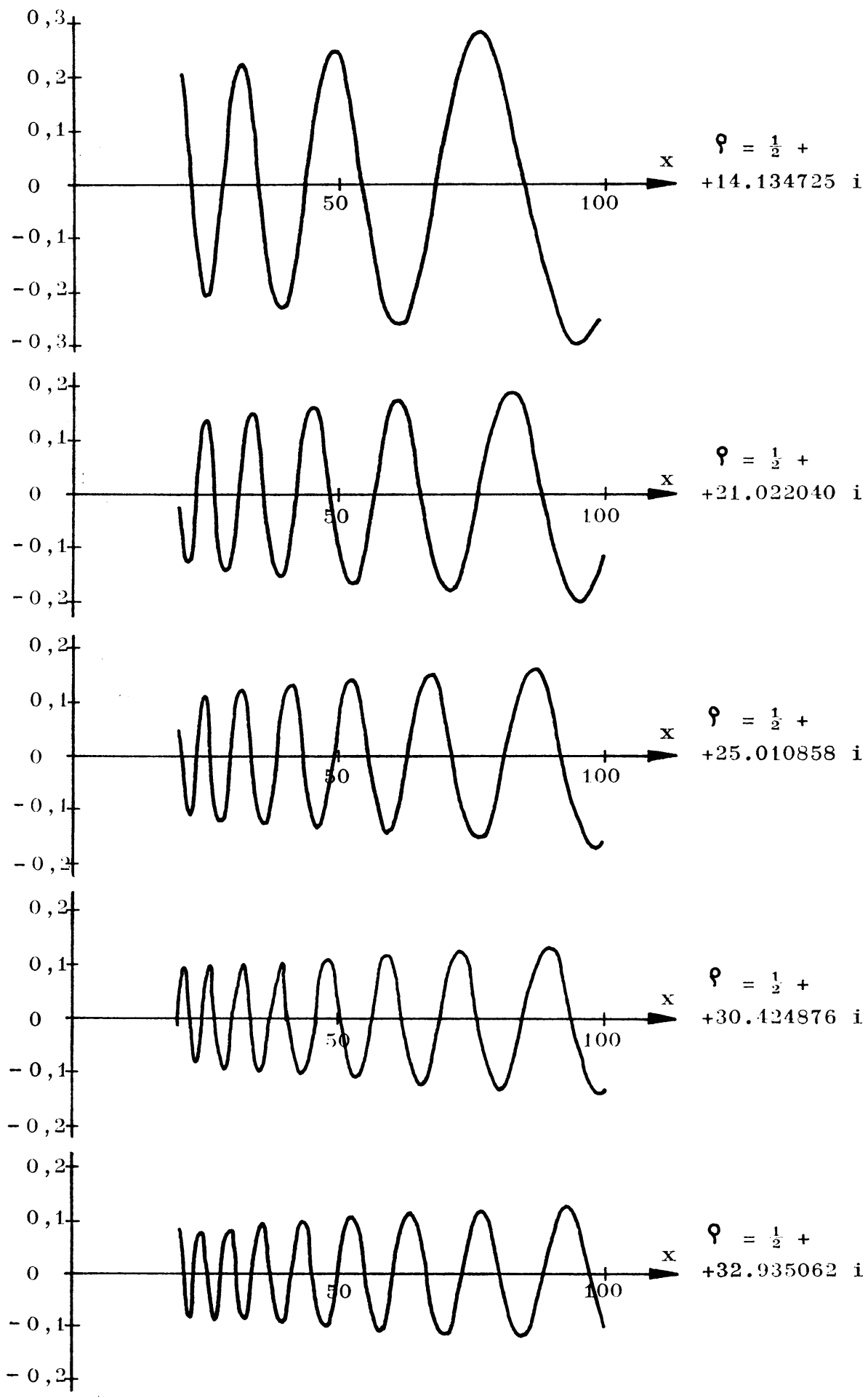

FIGURE 1. $y=-\frac{2 \sqrt{ } x}{|\rho| \log x} \cdot \cos (\alpha \cdot \log x-\arg \rho), \quad \rho=\frac{1}{2}+i \alpha$ 


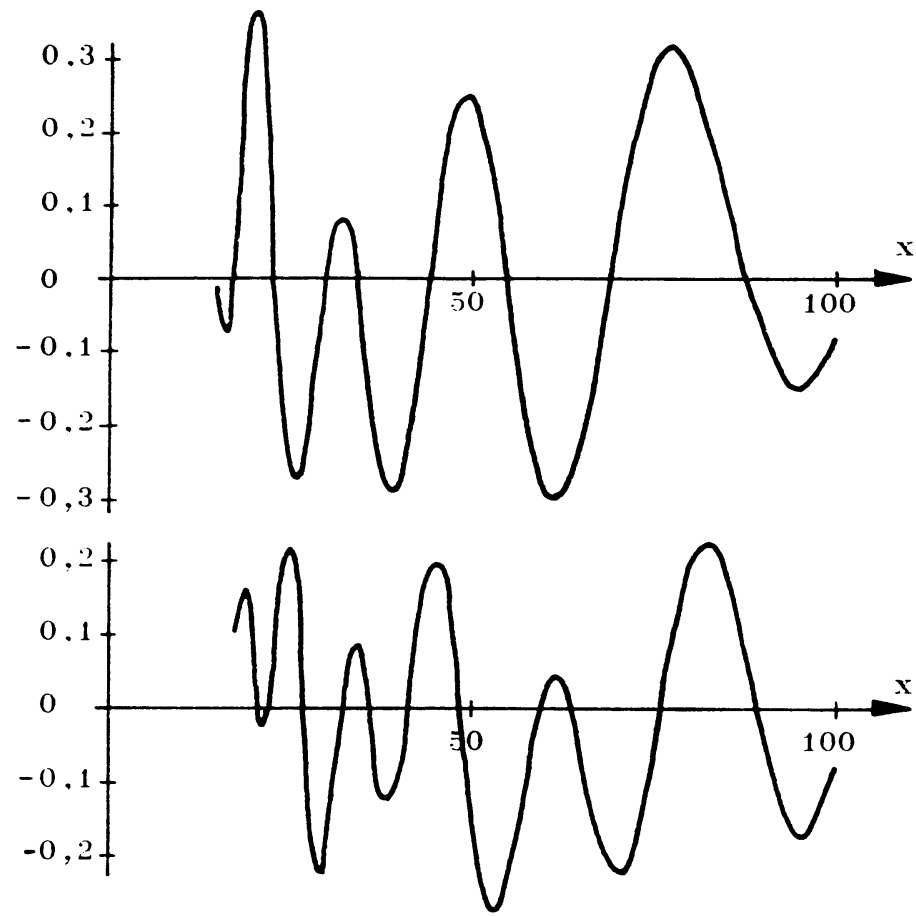

$$
\mathrm{T}_{1}(\mathrm{x})
$$

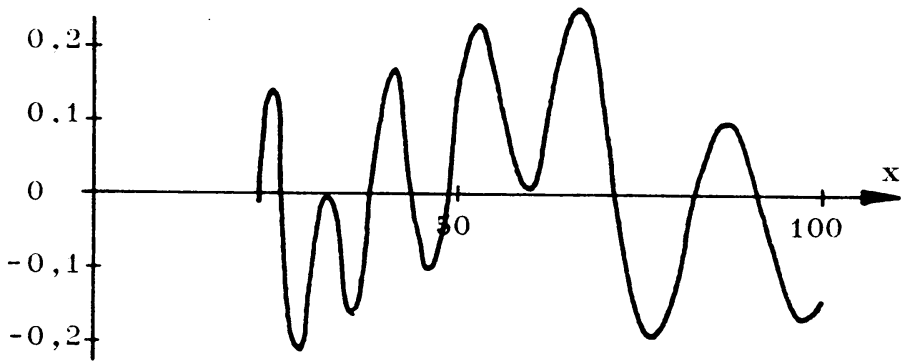

$$
\mathrm{T}_{2}(\mathrm{x})
$$
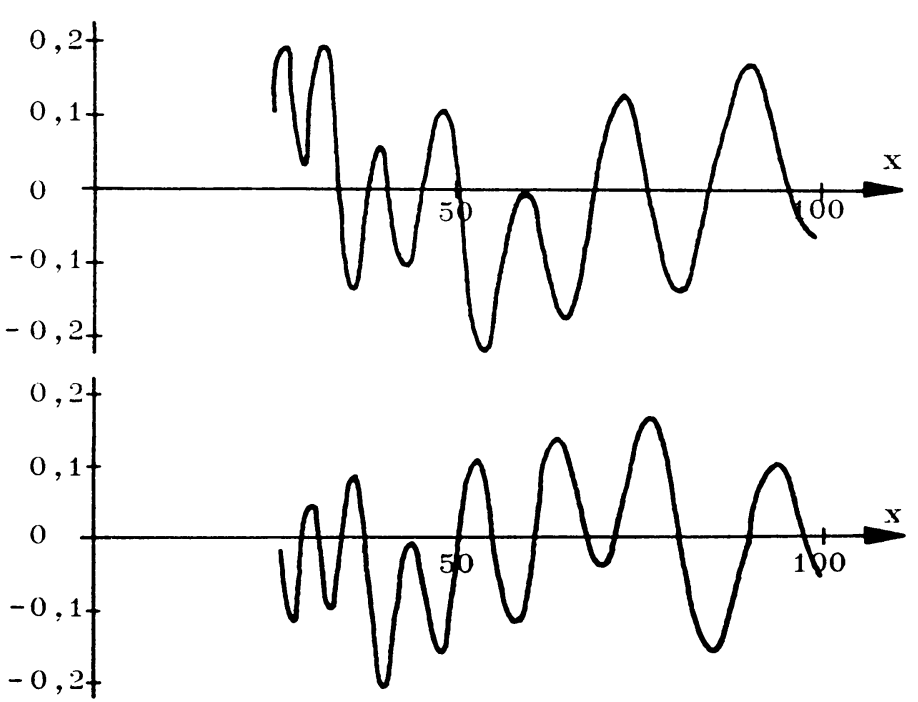

$\mathrm{T}_{5}(\mathrm{x})$

Figure 2. $T_{k}(x)$ for $k=1(1) 5$ (formula $\left.(14)\right)$ 
$\left\{\begin{array}{r}\pi(\mathrm{x}) \\ \cdots \cdots \mathrm{R}_{10}(\mathrm{x}) \\ -\mathrm{R}_{29}(\mathrm{x})\end{array}\right.$

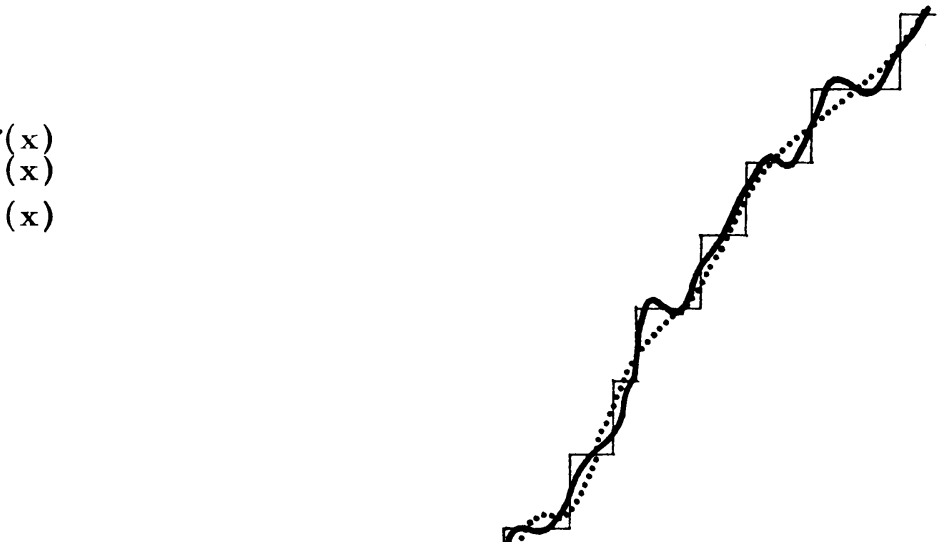

FIGURE 36

20
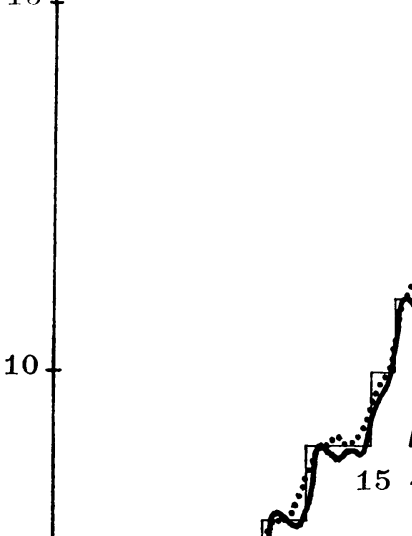

$\operatorname{li}_{15} \pi_{0}(x)$
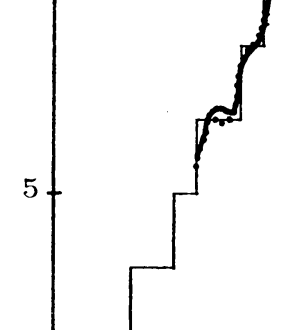

10

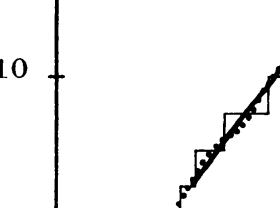

FIGURE 3a

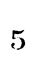

5
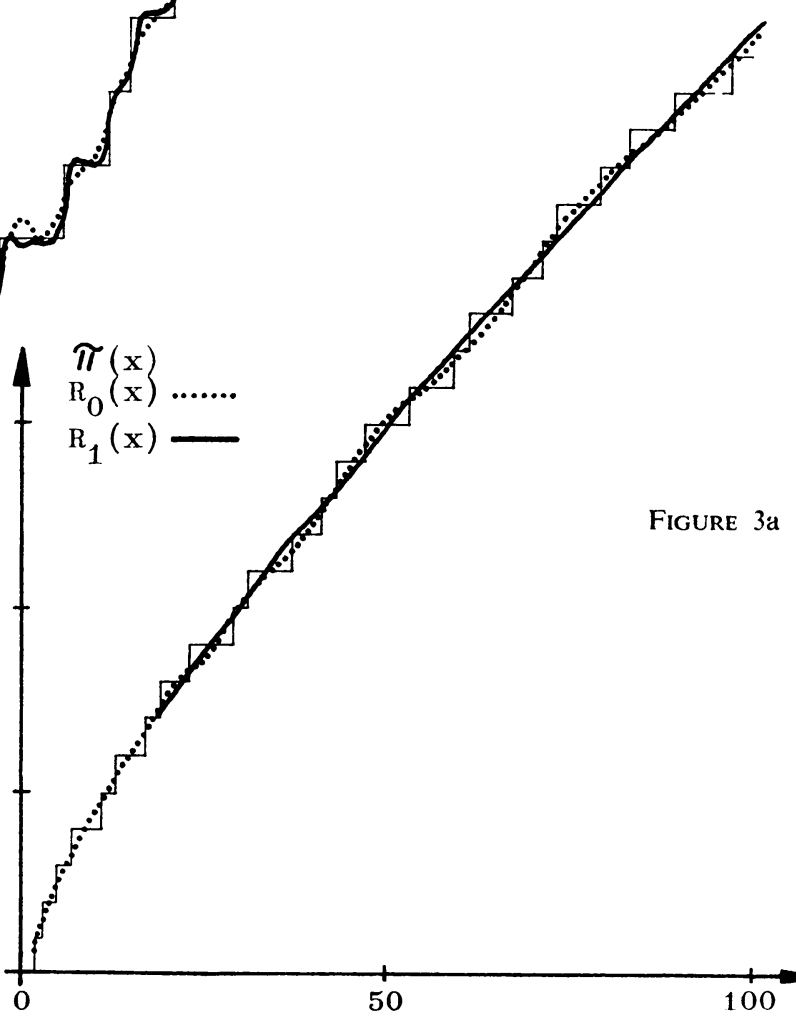


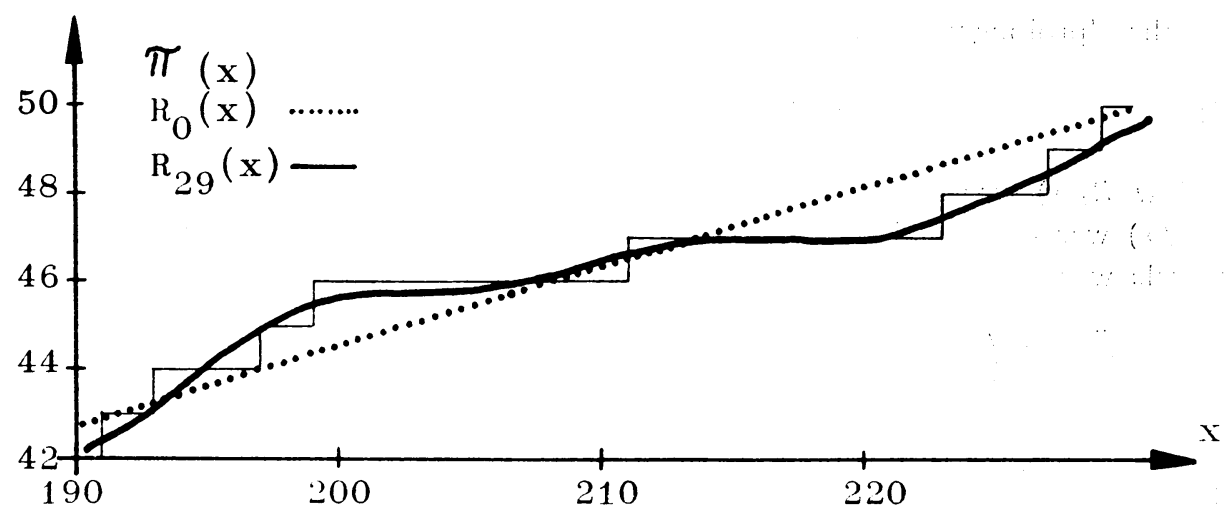

FIGURE 3c. $R_{0}(x)$ and $R_{29}(x)$ for $x=190.5(1) 230.5$

approximation that makes no use at all of the zeros, will exhaust the significant part of the function quite well, while quite a few zeros are needed in order to describe its more subtle properties. If the very large volume of computation needed to get at these better approximations is considered, they are indeed very slight improvements of (8).

As is natural to expect from (13), the larger zeros of $\zeta(s)$ influence $\pi_{0}(x)$ only for the larger values of $x$.

The Computations. Combining (5), (7), (14), and (15), we get for all $x<2^{N+1}$

$$
\pi_{0}(x)=R_{0}(x)+\sum_{\nu=1}^{\infty} T_{\nu}(x) .
$$

Taking only the first $k$ pairs of zeros of $\zeta(s)$, we get the following approximation $R_{k}(x)$ to $\pi_{0}(x)$ :

$$
\begin{aligned}
R_{k}(x)= & \sum_{n=1}^{N} \frac{\mu(n)}{n} \operatorname{li}\left(x^{1 / n}\right)+\sum_{n=1}^{N} \frac{\mu(n)}{n}\left(\int_{x^{1 / n}}^{\infty} \frac{d t}{\left(t^{2}-1\right) t \log t}-\log 2\right) \\
& -\sum_{\nu=1}^{k} \sum_{n=1}^{N} \frac{\mu(n)}{n}\left(1 \mathrm{i}\left(x^{\rho_{\nu} / n}\right)+\operatorname{li}\left(x^{\bar{\rho} / / n}\right)\right) .
\end{aligned}
$$

Using Gram's series (9) for

$$
\sum_{n=1}^{\infty} \mu(n) \operatorname{li}\left(x^{1 / n}\right) / n
$$

we get the following approximation to $R_{k}(x)$ :

$$
\begin{aligned}
R_{k}(x) \approx & R_{k}^{*}(x) \\
=1 & +\sum_{n=1}^{\infty} \frac{(\log x)^{n}}{n ! n \zeta(n+1)}+\sum_{n=1}^{N} \frac{\mu(n)}{n}\left(\int_{x^{1 / n}}^{\infty} \frac{d t}{\left(t^{2}-1\right) t \log t}-\log 2\right) \\
& -\sum_{n=1}^{k} \sum_{n=1}^{N} \frac{\mu(n)}{n}\left(\operatorname{li}\left(x^{\rho / n}\right)+1 \mathrm{i}\left(x^{\delta_{p} / n}\right)\right),
\end{aligned}
$$


with the "prolongation error"

$$
p(x, N)=-\sum_{N+1}^{\infty} \mu(n) \operatorname{li}\left(x^{1 / n}\right) / n .
$$

Now $R_{k}(x)$ was computed in the following way:

$R_{0}(x)$ was calculated by using Gram's series (9), and the sum containing the integrals was transformed as follows:

$$
\begin{aligned}
\sum_{n=1}^{N} & \frac{\mu(n)}{n}\left(\int_{x^{1 / n}}^{\infty} \frac{d t}{\left(t^{2}-1\right) t \log t}-\log 2\right) \\
= & \sum_{n=1}^{N} \frac{\mu(n)}{n} \int_{x^{1 / n}}^{e} \frac{d t}{\left(t^{2}-1\right) t \log t}+\sum_{n=1}^{N} \frac{\mu(n)}{n}\left(\int_{e}^{\infty} \frac{d t}{\left(t^{2}-1\right) t \log t}-\log 2\right) \\
& \left.\quad \text { (with the substitution } t=e^{n}\right) \\
= & \sum_{n=1}^{N} \frac{\mu(n)}{n} \int_{(\log x) / n}^{1} \frac{d u}{u\left(e^{2 u}-1\right)}+\sum_{n=1}^{N} \frac{\mu(n)}{n}\left(\int_{1}^{\infty} \frac{d u}{u\left(e^{2 u}-1\right)}-\log 2\right) .
\end{aligned}
$$

Introducing the partial fractions expansion of

$$
1 /\left(e^{z}-1\right)=1 / z-\frac{1}{2}+2 z \sum_{k=1}^{\infty}\left(z^{2}+4 \pi^{2} k^{2}\right)^{-1},
$$

the first sum in (21) becomes

$$
\begin{aligned}
\sum_{n=1}^{N} \frac{\mu(n)}{n} \int_{(\log x) / n}^{1}\left(\frac{1}{2 u^{2}}-\frac{1}{2 u}+\sum_{k=1}^{\infty} \frac{1}{u^{2}+\pi^{2} k^{2}}\right) d u \\
=\sum_{n=1}^{N} \frac{\mu(n)}{n}\left[-\frac{1}{2 u}-\frac{1}{2} \log u+\sum_{k=1}^{\infty} \frac{1}{\pi k} \arctan \frac{u}{\pi k}\right]_{(\log x) / n}^{1} \\
=\sum_{n=1}^{N} \frac{\mu(n)}{n}\left(-\frac{1}{2}+\frac{n}{2 \log x}+\frac{1}{2} \log \log x-\frac{1}{2} \log n\right. \\
\left.\quad+\sum_{k=1}^{\infty} \frac{1}{\pi k}\left(\arctan \frac{1}{\pi k}-\arctan \frac{\log x}{n \pi k}\right)\right) \\
=\frac{1}{2 \log x} \sum_{n=1}^{x} \mu(n)+\sum_{n=1}^{N} \frac{\mu(n)}{n}\left(-\frac{1}{2}+\frac{1}{2} \log \log x+\sum_{k=1}^{\infty} \frac{1}{\pi k} \arctan \frac{1}{\pi k}\right) \\
\quad-\frac{1}{2} \sum_{n=1}^{N} \frac{\mu(n) \log n}{n}-\left(\sum_{1}^{\infty}-\sum_{N+1}^{\infty}\right) \frac{\mu(n)}{n} \sum_{k=1}^{\infty} \frac{1}{\pi k} \arctan \frac{\log x}{n \pi k} .
\end{aligned}
$$

The double series $\sum_{1}^{\infty} \sum_{1}^{\infty}$ can be explicitly summed:

$$
\sum_{n=1}^{\infty} \sum_{k=1}^{\infty} \frac{\mu(n)}{n \pi k} \arctan \frac{\log x}{n \pi k}=\frac{1}{\pi} \sum_{m=1}^{\infty} \sum_{d \mid m} \frac{\mu(d)}{m} \arctan \frac{\log x}{\pi m} .
$$

Now, since

$$
\begin{aligned}
\sum_{d \mid m} \mu(d) & =0, \quad \text { if } \quad m \geqq 2 \\
& =1, \quad \text { if } \quad m=1,
\end{aligned}
$$




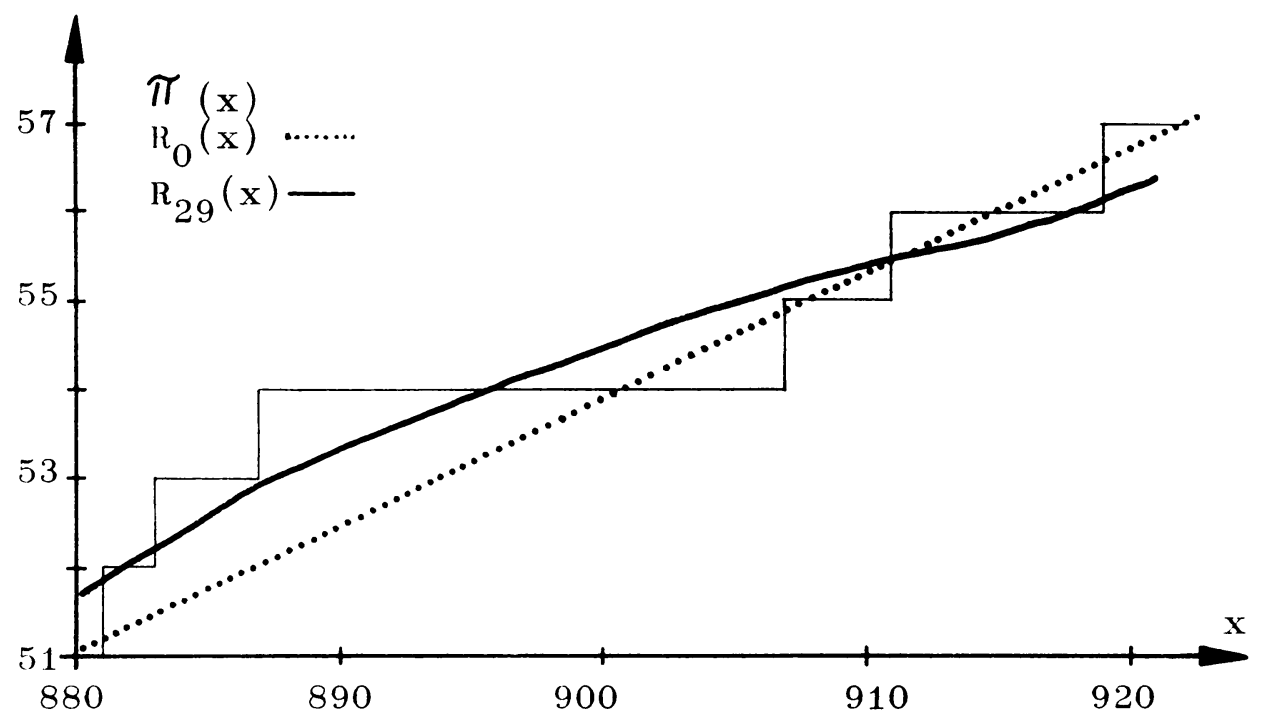

FIGURE 3d. $R_{0}(x)$ and $R_{29}(x)$ for $x=879.5(1) 920.5$

(24) reduces to only the one term, corresponding to $m=1$ :

$$
\sum_{n=1}^{\infty} \sum_{k=1}^{\infty}=\frac{1}{\pi} \arctan \frac{\log x}{\pi}=\frac{1}{2}-\frac{1}{\pi} \arctan \frac{\pi}{\log x} .
$$

We thus get

$$
\begin{aligned}
\sum_{n=1}^{N} \frac{\mu(n)}{n} & \left(\int_{x^{1 / n}}^{\infty} \frac{d t}{\left(t^{2}-1\right) t \log t}-\log 2\right) \\
= & \frac{1}{2 \log x} \sum_{n=1}^{N} \mu(n)+\frac{1}{\pi} \arctan \frac{\pi}{\log x}-\frac{1}{2} \sum_{n=1}^{N} \frac{\mu(n) \log n}{n}-\frac{1}{2} \\
& +\left(\int_{1}^{\infty} \frac{d u}{u\left(e^{2 u}-1\right)}-\log 2-\frac{1}{2}+\frac{1}{2} \log \log x+\sum_{k=1}^{\infty} \frac{1}{\pi k} \arctan \frac{1}{\pi k}\right) \sum_{n=1}^{N} \frac{\mu(n)}{n} \\
\text { (27) } \quad & +\sum_{N+1}^{\infty} \frac{\mu(n)}{n} \sum_{k=1}^{\infty} \frac{1}{\pi k} \arctan \frac{\log x}{n \pi k} \\
= & \frac{1}{2 \log x} \sum_{n=1}^{N} \mu(n)+\frac{1}{\pi} \arctan \frac{\pi}{\log x}-\frac{1}{2} \sum_{n=1}^{N} \frac{\mu(n) \log n}{n}-\frac{1}{2} \\
& +\left(\frac{1}{2} \log \log x-0.976904\right) \sum_{n=1}^{N} \frac{\mu(n)}{n} \\
& +\sum_{N+1}^{\infty} \frac{\mu(n)}{n} \sum_{k=1}^{\infty} \frac{1}{\pi k} \arctan \frac{\log x}{n \pi k} .
\end{aligned}
$$

Here the integral has been calculated as

$$
\begin{aligned}
\int_{1}^{\infty} \frac{d u}{u\left(e^{2 u}-1\right)} & =\int_{2}^{\infty} \frac{d t}{t\left(e^{t}-1\right)}=\sum_{k=1}^{\infty} \int_{2}^{\infty} e^{-k t} d t / t \\
& =\sum_{k=1}^{\infty} \int_{2 k}^{\infty} e^{-w} d w / w=\sum_{k=1}^{\infty} E_{1}(2 k)=0.0530823
\end{aligned}
$$


and the sum $\sum_{1}^{\infty}(1 / \pi k) \arctan (1 / \pi k)$ as

$$
\begin{aligned}
\sum_{k=1}^{\infty} \frac{1}{\pi k} \arctan \frac{1}{\pi k} & =\sum_{k=1}^{\infty} \frac{1}{\pi k}\left(\frac{1}{\pi k}-\frac{1}{3 \pi^{3} k^{3}}+\frac{1}{5 \pi^{5} k^{5}}-\cdots\right) \\
& =\frac{\zeta(2)}{\pi^{2}}-\frac{\zeta(4)}{3 \pi^{4}}+\frac{\zeta(6)}{5 \pi^{6}}-\cdots=0.1631606 .
\end{aligned}
$$

Letting $N$ tend to $\infty$, and using the known sums of the convergent series

$$
\begin{gathered}
\sum_{n=1}^{\infty} \mu(n) / n=0, \\
\sum_{n=1}^{\infty} \frac{\mu(n) \log n}{n}=-1,
\end{gathered}
$$

we finally get for the sum with the integrals in (19)

$$
\begin{aligned}
\sum_{n=1}^{N} \frac{\mu(n)}{n}\left(\int_{x^{1 / n}}^{\infty} \frac{d t}{\left(t^{2}-1\right) t \log t}\right. & -\log 2) \\
= & \frac{1}{2 \log x} \sum_{n=1}^{N} \mu(n)+\frac{1}{\pi} \arctan \frac{\pi}{\log x}+\epsilon(x, N),
\end{aligned}
$$

where

$$
\begin{aligned}
\epsilon(x, N)= & -\left(\frac{1}{2} \log \log x-0.976904\right) \sum_{N+1}^{\infty} \frac{\mu(n)}{n}+\frac{1}{2} \sum_{N+1}^{\infty} \frac{\mu(n) \log n}{n} \\
& +\sum_{N+1}^{\infty} \frac{\mu(n)}{n} \sum_{k=1}^{\infty} \frac{1}{\pi k} \sum_{m=1}^{\infty} \frac{(-1)^{m-1}(\log x)^{2 m-1}}{(\pi k n)^{2 m-1}(2 m-1)} .
\end{aligned}
$$

Thus $\epsilon \rightarrow 0$, as $N \rightarrow \infty$. This simple result of the inversion of the integral term in Riemann's formula (4) does not seem to have been noticed earlier.

Thus $R_{0}(x)$ was calculated using Gram's series and the formula (32) after omission of the term $\epsilon(x, N)$.

The integral logarithms for the complex arguments were calculated by using the well-known continued fraction expansion of $\operatorname{Ei}(z)$, which leads to

$$
\operatorname{li}\left(e^{z}\right)=\frac{e^{z} \mid}{\mid z}-\frac{1 \mid}{\mid 1}-\frac{1 \mid}{\mid z}-\frac{2 \mid}{\mid 1}-\frac{2 \mid}{\mid z}-\cdots .
$$

In the vicinity of $z=0$, however, the power series

$$
\operatorname{li}\left(e^{z}\right)=\gamma+\log (-z)+\sum_{n=1}^{\infty} \frac{z^{n}}{n ! n},
$$

where $|\arg (-z)|<\pi$, and $\gamma$ is Euler's constant, instead was used.

Choice of $N$. By using (32) as an approximation to (27), we are making the prolongation error

$$
\begin{aligned}
\epsilon(x, N)= & \left(\frac{1}{2} \log \log x-0.976904\right) \sum_{n=1}^{N} \frac{\mu(n)}{n}-\frac{1}{2}\left(1+\sum_{n=1}^{N} \frac{\mu(n) \log n}{n}\right) \\
& +\sum_{N+1}^{\infty} \frac{\mu(n)}{n} \sum_{k=1}^{\infty} \frac{1}{\pi k} \sum_{m=1}^{\infty} \frac{(-1)^{m-1}(\log x)^{2 m-1}}{(\pi k n)^{2 m-1}(2 m-1)},
\end{aligned}
$$

where the MacLaurin's expansions of the arctangent functions converge because 
of the assumption $x<2^{N+1}$, which we always make in order to be able to use (7). The triple sum in (35) can be reduced to

$$
\sum_{m=1}^{\infty} \frac{(-1)^{m-1}(\log x)^{2 m-1}}{(2 m-1) \pi^{2 m}} \zeta(2 m) \sum_{n=N+1}^{\infty} \frac{\mu(n)}{n^{2 m}} .
$$

We thus see that the series contains the sums $g_{k}=\sum_{N+1}^{\infty} \mu(n) n^{-k}$. It is thus advantageous to choose such a value of $N$ that the sums $g_{k}$ become comparatively small. It also is advantageous to have $\sum_{1}^{N} \mu(n)=-2$ at the same time, since (32) then has the order of magnitude only $=O\left((\log x)^{-3}\right)$ instead of $O\left((\log x)^{-1}\right)$. This turns out to be the case for (among some other small values of $N$ ) $N=154$. In our computations we have chosen this value of $N$ in (14) and (19). To estimate $g_{k}=\sum_{155}^{\infty} \mu(n) n^{-k}$. we calculated $g_{k}$ as

$$
g_{k}=1 / \zeta(k)-\sum_{1}^{154} \mu(n) n^{-k} \text { for } k=2(1) 9,
$$

using floating point, double precision $(84+12$ bits) arithmetic. For $k \geqq 10$ we calculated $g_{k}$ as

$$
g_{k}=\sum_{1.5 .5}^{86.5} \mu(n) n^{-k} \quad \text { for } \quad k=10(1) 20,
$$

where the absolute value of the remainder

$$
\left|\sum_{866}^{\infty} \mu(n) n^{-k}\right|<\sum_{866}^{\infty} n^{-k}<\int_{865}^{\infty} x^{-k} d x=\frac{865^{-(k-1)}}{k-1} .
$$

The values of $g_{k}$ are given in the following Table 1. We also give in the table some simple inequalities for $\left|g_{k}\right|$, which are used in the subsequent analysis.

Analysis of the Prolongation Errors. The error (20),

$$
p=p(x, N)=-\sum_{N+1}^{\infty} \mu(n) \operatorname{li}\left(x^{1 / n}\right) / n,
$$

can be estimated by use of the well-known expansion

$$
\operatorname{li}\left(x^{1 / n}\right)=\operatorname{Ei}((\log x) / n)=\gamma+\log \left(\frac{1}{n} \log x\right)+\sum_{k=1}^{\infty} \frac{(\log x)^{k}}{n^{k} k \cdot k !} .
$$

Thus.

$$
p=-(\gamma+\log \log x) \sum_{N+1}^{\infty} \mu(n) / n+\sum_{N+1}^{\infty} \frac{\mu(n) \log n}{n}-\sum_{k=1}^{\infty} \frac{(\log x)^{k}}{k \cdot k !} \sum_{n=N+1}^{\infty} \frac{\mu(n)}{n^{k+1}} .
$$

Putting $N=154$, we get

$$
p=-(\gamma+\log \log x) g_{1}-0.000641176-\sum_{k=1}^{\infty}(\log x)^{k} g_{k+1} /(k \cdot k !) .
$$

The error $\epsilon(x, N)$, defined in (35), equals

$$
\begin{aligned}
\epsilon(x, N)= & \left(\frac{1}{2} \log \log x-0.976904\right)\left(-g_{1}\right)-0.000320588 \\
& +\sum_{m=1}^{\infty} \frac{(-1)^{m-1}(\log x)^{2 m-1} \zeta(2 m)}{(2 m-1) \pi^{2 m}} g_{2 m} .
\end{aligned}
$$


TABLE $1 . \quad g_{k}=\sum_{155}^{\infty} \mu(n) n^{-k}$

\begin{tabular}{cc}
$k$ & $g_{k}$ \\
\hline 1 & $-1.44447 \cdot 10^{-4}$ \\
2 & $-1.114766 \cdot 10^{-6}$ \\
4 & $-3.479177 \cdot 10^{-9}$ \\
5 & $3.135720 \cdot 10^{-11}$ \\
6 & $7.197512 \cdot 10^{-13}$ \\
7 & $8.886832 \cdot 10^{-15}$ \\
8 & $8.912980 \cdot 10^{-17}$ \\
9 & $7.99391 \cdot 10^{-19}$ \\
10 & $6.673 \cdot 10^{-21}$ \\
11 & $5.296 \cdot 10^{-23}$ \\
12 & $4.0468 \cdot 10^{-25}$ \\
13 & $3.00369 \cdot 10^{-27}$ \\
14 & $2.17850 \cdot 10^{-29}$ \\
15 & $1.55080 \cdot 10^{-31}$ \\
16 & $1.08722 \cdot 10^{-33}$ \\
17 & $7.52644 \cdot 10^{-36}$ \\
18 & $5.15555 \cdot 10^{-38}$ \\
19 & $3.50029 \cdot 10^{-40}$ \\
20 & $2.35868 \cdot 10^{-42}$ \\
$k \geqq 20$ & $1.57927 \cdot 10^{-44}$ \\
$k \geqq 1$ & $<154^{1-k} /(k-1)$ \\
\hline
\end{tabular}

Thus, the total error, $p+\epsilon$, adds up to

$$
\begin{aligned}
p+\epsilon= & -g_{1}(1.5 \log \log x+\gamma-0.976904)-0.000961764 \\
& -\frac{5}{6} g_{2} \log x-\frac{1}{4} g_{3}(\log x)^{2}-\frac{8}{135} g_{4}(\log x)^{3} \\
& -\sum_{k=4}^{\infty}(\log x)^{k} g_{k+1} /(k \cdot k !)+\sum_{m=3}^{\infty} \frac{(-1)^{m-1}(\log x)^{2 m-1} \zeta(2 m)}{(2 m-1) \pi^{2 m}} g_{2 m} \\
= & 1.44447 \cdot 10^{-4}(1.5 \log \log x-0.399688) \\
& -0.000961764+9.28972 \cdot 10^{-7} \log x \\
& +8.69794 \cdot 10^{-10}(\log x)^{2}-1.85820 \cdot 10^{-12}(\log x)^{3}+R .
\end{aligned}
$$

For all $x$ between 2 and $2^{155}$, this expression is smaller than

$$
1.1 \cdot 10^{-4}+|R| \text {. }
$$

$R$ can be estimated using the first inequality in Table 1 :

$$
\begin{aligned}
\sum_{k=1}^{\infty}(\log x)^{k} g_{k+1} /(k \cdot k !) & \leqq \\
& \left.<\sum_{k=4}^{20}+\sum_{k=21}^{\infty}\right) 107.44^{k}\left|g_{k+1}\right| /(k \cdot k !) \\
& <1.26 \cdot 10^{-6}+\sum_{k=21}^{\infty}(107.44 / 154)^{k} /\left(k^{2} k !\right)<1.3 \cdot 10^{-6} .
\end{aligned}
$$


Furthermore,

$$
\left|\sum_{m=3}^{\infty} \frac{(-1)^{m-1}(\log x)^{2 m-1} \zeta(2 m)}{(2 m-1) \pi^{2 m}} g_{2 m}\right|<\frac{\zeta(6)}{5 \pi} \sum_{m=3}^{\infty}\left(\frac{107.44}{154}\right)^{2 m-1} \cdot \frac{7.7}{154}<1.9 \cdot 10^{-5} .
$$

Combining (45), (46), and (47), we get the following bound on the total error

$$
|p+\epsilon|<1.1 \cdot 10^{-4}+0.032 \cdot 10^{-4}<1.2 \cdot 10^{-4},
$$

for all $x$ between 2 and $2^{155}$.

The Difference Between $R_{k}(x)$ and $\pi_{0}(x)$. By comparing the computed values $R_{k}^{*}(x)$ with those of $\pi_{0}(x)$, we can, since we know the upper bound $1.2 \cdot 10^{-4}$ on the error in $R_{k}^{*}(x)$, tell how good an approximation $R_{k}(x)$ is to $\pi_{0}(x)$. Since $R_{k}(x)$ is very nearly $=R_{k}^{*}(x)$ for $x<2^{155}$, the difference, $R_{k}(x)-\pi_{0}(x)$, can be calculated with good accuracy as $R_{k}^{*}(x)-\pi_{0}(x)$. This difference is easy to see in Figs. 3a-d for certain intervals and certain values of $k$.

We calculated $R_{k}^{*}(x)$ for $k=0,1,2,3,4,5,10$, and 29 for $x=2(1) 100$, for $x=p \cdot 10^{a}$, where $p=1(1) 9$ and $q=2(1) 5$, and for $x=10^{6}$. Furthermore, we made, as has already been mentioned, studies for $x$-values in some specially chosen intervals, namely for $x=190.5(1) 230.5$ and for $x=879.5(1) 920.5$. Because of the large volume of computation needed, we did not calculate more values and thus could make a systematic study of $R_{k}(x)-\pi_{0}(x)$ only up to $x=100$ and in the special intervals $(190.5,230.5)$ and $(879.5,920.5)$. For $x \leqq 100$, we give the results in Table 2 , which contains those values of $x$ for which $\left|R_{k}^{*}(x)-\pi_{0}(x)\right|$ takes a larger maximum value than for any $t \leqq x$. We also give the corresponding values of $S=R_{k}^{*}(x)-\pi_{0}(x)$.

Because we did not calculate the functions with a much smaller step in $x$, say 0.01 , we obviously have not always "hit" on the true maximum values of $|S|$, but only

\begin{tabular}{|c|c|c|c|c|c|c|c|c|c|c|c|c|c|}
\hline \multicolumn{2}{|c|}{$k=0$} & \multicolumn{2}{|c|}{$k=1$} & \multicolumn{2}{|c|}{$k=2$} & \multicolumn{2}{|c|}{$k=3$} & \multicolumn{2}{|c|}{$k=4$} & \multicolumn{2}{|c|}{$k=5$} & \multicolumn{2}{|c|}{$k=10$} \\
\hline$x$ & $S$ & $x$ & $S$ & $x$ & $S$ & $x$ & $S$ & $x$ & $S$ & $x$ & $S$ & $x$ & $S$ \\
\hline 2 & 0.03 & 2 & 0.05 & 2 & -0.09 & 2 & 0.11 & 2 & -0.06 & 2 & -0.02 & 2 & -0.15 \\
\hline 4 & 0.07 & 4 & -0.11 & 3 & 0.10 & 18 & 0.15 & 3 & -0.11 & 3 & -0.07 & 19 & -0.17 \\
\hline 7 & -0.14 & 5 & 0.12 & 10 & 0.14 & 19 & 0.22 & 4 & 0.16 & 5 & -0.08 & 33 & 0.27 \\
\hline 8 & -0.27 & 7 & -0.16 & 18 & 0.22 & 24 & -0.29 & 23 & -0.17 & 9 & 0.14 & 48 & -0.32 \\
\hline 10 & 0.43 & 14 & -0.22 & 24 & -0.43 & 38 & -0.31 & 24 & -0.31 & 15 & -0.17 & 68 & -0.56 \\
\hline 20 & -0.55 & 16 & 0.23 & 58 & 0.47 & 40 & 0.39 & 40 & 0.43 & 21 & 0.19 & - & 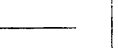 \\
\hline 28 & 0.60 & 23 & -0.26 & 62 & -0.62 & 52 & 0.52 & 58 & 0.47 & 36 & 0.21 & $k$ & $=29$ \\
\hline 36 & 0.61 & 24 & -0.63 & & & 62 & -0.54 & 62 & -0.62 & 38 & -0.40 & $x$ & $S$ \\
\hline 58 & 0.75 & & -0.66 & & & 74 & -0.73 & & & 52 & 0.45 & & - \\
\hline 74 & -0.76 & 96 & 0.68 & & & & & & & 62 & -0.51 & 2 & 0.21 \\
\hline 96 & 0.82 & & & & & & & & & & & 31 & -0.23 \\
\hline & & & & & & & & & & & & 78 & 0.25 \\
\hline & & & & & & & & & & & & 96 & 0.29 \\
\hline
\end{tabular}

TABLE 2. $x$ and $S=R_{k}^{*}(x)-\pi_{0}(x)$ when $|S|$ has a large max. 
on neighbouring values to the extreme values. This explains why in Table $2 R_{2}^{*}(x)$ seems to be an equally good approximation as $R_{4}^{*}(x)$ to $\pi_{0}(x)$, or why $R_{10}^{*}(x)$ even seems to be a little bit worse than $R_{5}^{*}(x)$. Also, for a fixed $x$, we occasionally may get worse approximations, when we raise $k$, namely if the error $R_{i}^{*}(x)-\pi_{0}(x)$ happens to be exactly equal to 0 for some small value of $j$. However, the trend is towards smaller deviations "in mean" between $R_{k}^{*}(x)$ and $\pi_{0}(x)$ as $k$ grows. This is well supported by the small values obtained for $k=29$.

For $x$ in the intervals $(190.5,230.5)$ and $(879.5,920.5)$, we give $\max \left|R_{k}(x)-\pi_{0}(x)\right|$ in Table 3.

The trend towards smaller deviations with growing $k$ is the same as in Table 2.

From the Tables 2 and 3 we infer that $R_{29}(x)$ approximates $\pi_{\rho}(x)$ with an error of at most a few units for $x \leqq 1000$.

Some Earlier Computations. On checking the program-routines for $\operatorname{li}(x)$ and $R(x)$, the authors calculated the values of these functions for $x=p \cdot 10^{4}$, where $p=10(1) 99$ and $q=0(1) 10$. Some of these values are found in [5] [8]. On this occasion some minor errors in the previous work were detected. First, we note that different authors define $\operatorname{li}(x)$ and $R(x)$ in a slightly different way. Thus,

\begin{tabular}{|c|c|c|}
\hline \multirow{3}{*}{ in [6] equals } & $\operatorname{li}(x)$ & $R(x)$ \\
\hline & - & ours -1 \\
\hline & $\begin{array}{l}\text { ours in all } \\
\text { computations }\end{array}$ & \\
\hline in [5], [7], and [8] equals & $\left\{\begin{array}{l}\text { ours-li( } 2 \text { ) in } \\
\text { defining relation }\end{array}\right.$ & ours \\
\hline
\end{tabular}

Taking these differences into account $(\operatorname{li}(2)=1.045)$, we find that in [5]

$R(x)$ was given 1 unit too high for $x \cdot 10^{-6}=0.75,1$, and 2.4,

$R(x)$ was given 1 unit too low for $x \cdot 10^{-6}=4.7$, and that

li $(x)$ was given 1 unit too high for $x \cdot 10^{-6}=0.65,1.2,4.4,8.2$, and 8.8 .

The values for $x \cdot 10^{-6}=1.05(0.1) 9.95$ were not checked by us. Curiously enough, the values of $R(x)$ for $x \cdot 10^{-6}=1,2.4$, and 4.7, are correctly given in [6], which is published much earlier than [5].

In [7], the values of $R(x)$ are given 1 unit too low for

$$
x \cdot 10^{-6}=20,25,33,40, \text { and } 90 .
$$

$R\left(37 \cdot 10^{6}\right)$ is given 4 units too low. $\operatorname{li}\left(10^{8}\right)$ is given 1 unit too high, and $\operatorname{li}\left(9 \cdot 10^{7}\right)$ is 457 units too low.

In [8], finally, where we have recalculated all given values of li $(x)$ and $R(x)$, we found only one minor mistake. $R\left(83 \cdot 10^{7}\right)$ is given as 42608308 , where we found $42608307 \cdot 499944$.

TABLE 3. $\max _{x \in \boldsymbol{I}}\left|R_{k}(x)-\pi_{0}(x)\right|$

\begin{tabular}{ccccccccc}
\hline$I$ & $k=0$ & $k=1$ & $k=2$ & $k=3$ & $k=4$ & $k=5$ & $k=10$ & $k=29$ \\
\hline$(190.5,230.5)$ & 1.58 & 1.38 & 1.21 & 1.32 & 1.21 & 1.17 & 0.89 & 0.75 \\
$(879.5,920.5)$ & 1.91 & 2.50 & 2.02 & 2.05 & 1.90 & 1.79 & 1.34 & 1.19 \\
\hline
\end{tabular}


Acknowledgments. The authors want to thank Prof. Germund Dahlquist for stimulating discussions and valuable advice, especially in connexion with the "prolongation error", and the Royal Institute of Technology for furnishing computing time.

Department of Information Processing

Royal Institute of Technology

S-100 44 Stockholm 70

Sweden

Marknadsvägen 229

S-183 34 Täby

Sweden

1. B. Riemann, "Über die Anzahl der Primzahlen unter einer gegebenen Grösse," Monatsh. Königl. Preuss. Akad. Wiss. Berlin, 1859, pp. 671-680; see also: B. Riemann, Gesammelte mathematische Werke und wissenschaftlicher Nachlass, reprint, Dover, New York, 1953, pp. 145-155. MR 14, 610.

2. H. von Mangoldt, "Zu Riemanns Abhandlung "über die Anzahl der Primzahlen unter einer gegebenen Grösse'," J. Reine Angew. Math., v. 114, 1895, pp. 255-305.

3. E. LANDAU, Handbuch der Lehre von der Verteilung der Primzahlen, 2nd ed., Chelsea, New York, 1953, pp. 333-370. MR 16, 904.

4. A. E. Ingham, The Distribution of Prime Numbers, Cambridge Tracts in Math. and Math. Phys., no. 30, Stechert-Hafner, New York, 1964. MR 32 \#2391. pp. IX $-\mathrm{X}$

5. D. N. LeHMER, List of Prime Numbers from 1 to 10,006,721, Stechert-Hafner, New York, 1956.

6. J. P. Gram, "Rapport sur quelques calculs entrepris par M. Bertelsen et concernant les nombres premiers," Acta Math., v. 17, 1893, pp. 301-314.

7. D. H. LeHMER, "On the exact number of primes less than a given limit," Illinois J. Math.. v. 3, 1959, pp. 381-388. MR $21 \# 5613$.

8. D. C. MAPES, "Fast method for computing the number of primes less than a given limit." Math. Comp., v. 17, 1963, pp. 179-185. MR $28 \# 1731$. 